\title{
LOCALIZATION METHOD FOR LOW POWER CONSUMPTION SYSTEMS
}

\author{
D. F. Larios, J. Barbancho, F. J. Molina and C. León \\ Department of Electronic Technology, Escuela Politécnica Superior, University of Seville, Seville, Spain \\ dflarios@dte.us.es
}

\begin{abstract}
Keywords: $\quad$ Localization, WSN, Fuzzy system, RSSI, Centroid, CL.
Abstract: $\quad$ Locating nodes is a fundamental problem in wireless networks with hundreds of devices deployed in a wide area. This is especially relevant for mobile nodes. Wireless sensor nodes are usually powered by small batteries, solar panels or piezoelectric generators, so that, and consequently, power consumption is the main constraint to deal with. But classic localization techniques do not consider the problem of energy consumption as a key point. This paper presents a novel low power and range-free localization technique based on distributed fuzzy logic and cooperative processing among a set of fixed nodes and its neighbours. This feature permits better accuracy with less power consumption than most relevant localization techniques
\end{abstract}

\section{INTRODUCTION}

A wireless sensor network (WSN) consists of lots of small devices deployed in a physical environment for its study. Each node has special capabilities, such as wireless communications with its neighbours, sensing, data storage and processing.

WSNs have been widely used in many areas (Akyildiz et al., 2002), such as environmental monitoring (Yick et al., 2008), control (Riquelme et al., 2009), healthcare and medical research (Chung et al., 2008), national defense and military affairs (He et al., 2004) (Boukerche et al., 2008), etc.

For most of these applications, it is usually necessary to implement methods to estimate these positions. In many cases, this information gathered from the nodes is irrelevant without the knowledge of the associated position, for example in WSN applied to study wildfires (Antoine-Santoni et al., 2009). In other applications, the node position is in fact the required information (e.g vehicle tracking (Tubaishat et al., 2009)).

Most important constraints for the design and management of WSNs are data storage capability and processing, weight (especially for mobile devices), power consumption, cost, and radio coverage.

This paper presents LIS (Localization based on Intelligent System), a novel localization algorithm based on fuzzy logic processing. LIS is focused on the reduction of energy consumption.

The paper is organized as follows: section 2 sums up the state of the art about localization. Section 3 describes LIS. Section 4 shows the benefits of LIS in power consumption. Simulations and the LIS performance are summed up in section 5. Finally, in section 6 , we present concluding remarks and future work.

\section{LOCALIZATION TECHNIQUES}

In localization applications, there are two types of nodes:

- Anchor Nodes. Situated on fixed and known positions.

- Non-anchor Nodes (Tags). Nodes with unknown position. These nodes are usually called tags.

Localization algorithms presented in the literature can be classified into two categories, as given below:

- Range-based. These techniques estimate, point-to-point, the distance between each pair of nodes. With this information and using techniques, such as triangulation, the absolute position of the non-anchor nodes can be estimated. Generally, range-based techniques require additional hardware. The most common ones are Received Signal Strength Indication (RSSI) (Awad et al., 2007), Time Of Arrival (TOA) (Wu and Zhang, 2007) and Angle Of Arrival (AOA) (Rong and Sichitiu, 2006). 
- Range-free. The position of the non-anchor nodes is obtained from the beacons sent by the anchor nodes. Beacons contain information about area coverage membership or number of hops between devices. The most common range-free techniques are Centroid (CL) (Bulusu et al., 2000) and DV-Hop (Gao and Lei, 2010).

In general, the range-based ones offer good accuracy, but additional hardware is often needed. Therefore, the weight, the cost and the power consumption of the node devices increase and make these techniques unsuitable for the proposed application. RSSI range-based techniques are an exception to this because most of the current transceivers provide this measurement by default. However, RSSI techniques are very sensitive to noise and interferences. Figure 1 shows experiments to evaluate the relationship between RSSI and the distance in different situations: free-space without obstacles and long urban area with obstacles. The results are expressed in absolute value.

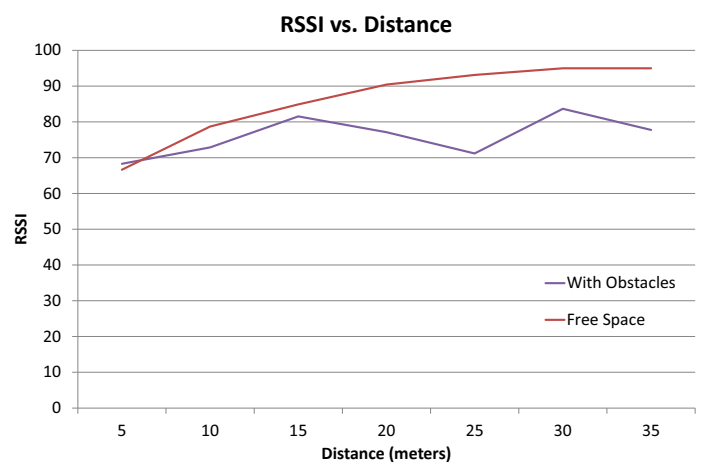

Figure 1: RSSI vs. distance.

The results with obstacles do not match with any valid mathematical model that could directly permit to obtain the distance, using only the RSSI. Obtaining models with the relationship between RSSI and distance is currently an important area of research. In any case, it is possible to obtain this model with a much studied environment. However, the main problem is to obtain the inverse of this relationship. In fact different distances produced the same RSSI value. Thus, even having a correct model of the environment and ideal situations, it is not possible to obtain the distance using only the RSSI information.

Instead of using a mathematical model, LIS proposes a fuzzy-logic-based system to derive the distance from RSSI level. This is more robust in noisy and complex scenarios. The use of computational intelligence in localization is not a novel idea, as could be seen in previous works, such as (Rajaee et al., 2008) that uses probabilistic neuronal networks, (Xiufang et al., 2008) that applies a fuzzy system and (Chiang and Wang, 2009) that uses fuzzy neurons. In general, all these are distributed algorithms that execute almost an important part of the localization algorithm over the non-anchor nodes. However, none of them consider the problem of power consumption in the non-anchor nodes. Moreover, the algorithms with Computational Intelligence generally track down the current positions based on the estimated position changes, needing an initialization of the non-anchor nodes. These systems fail if the tags (the animal) go out of the coverage of the WSN, and return into it after a while.

\section{LIS ALGORITHM}

Despite the fact that the range-free and range-based techniques have been extensively studied, nowadays there are some aspects that continue to be a challenge:

- The use of additional hardware or lots of beacons to increase power consumption.

- Fully centralized processing (i.e. on Base Stations) requires a large amount of messages. Conversely, processing in the tags' nodes reduces the battery of these devices significantly.

- Scalability. Many localization algorithms are hard to extend to the big sensor networks.

LIS has been especially designed to phase out all of the above mentioned problems. As a result, the proposed algorithm is scalable and the power consumption and network autonomy are optimized. As usual in a tracking system, the non-anchor nodes in LIS do not need to know their location, In this case, only the Base Station wants to know it. LIS combines: (1) a fuzzy system to estimate (actually to qualify) the distance between transmitter and receiver from RSSI measures, (2) a ubiquitous algorithm executed in receiver anchor nodes to determine relative positions to them, and (3) a cooperative algorithm to derive the most likely location running at the Base Station.

LIS consists of four stages:

S1. Anchor nodes wait for non-anchor node beacons.

S2. The tag node broadcasts a beacon.

S3. Receiver anchor nodes measure RSSI, and execute both, the fuzzification algorithm and the ubiquitous processing for relative and partial positioning.

S4. Anchor nodes send partial solutions to the Base Station, where the location is finally determined. 


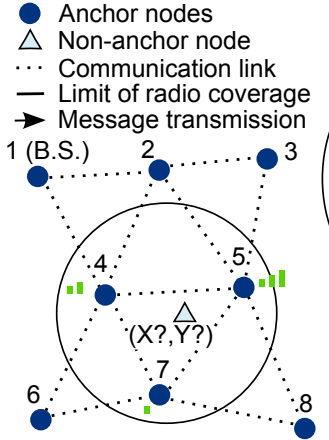

(a)

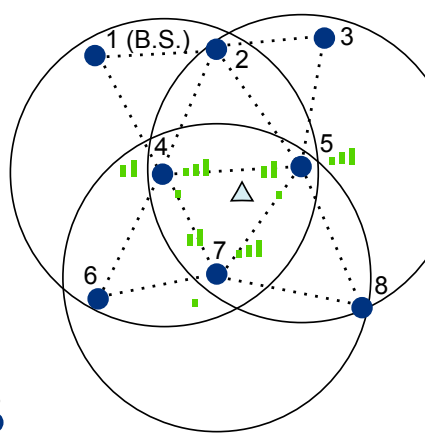

(b)

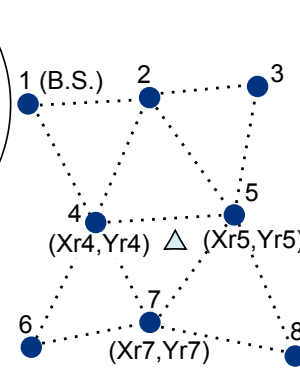

(c)

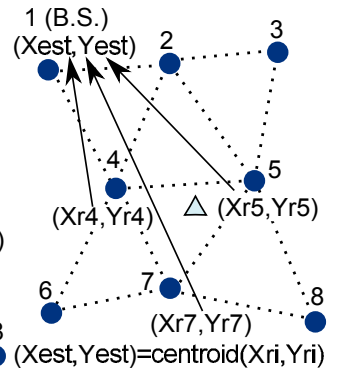

(d)

Figure 2: Steps of LIS algorithm.

Figure 2 illustrates these stages. When a non-anchor node broadcasts a beacon or any other sort of message, the localization process starts (figure 2.a). Just at that moment the receiver anchor nodes participate in the process. The rest of the nodes can switch off the radio transceiver or hold in it a low power state.

\subsection{Ubiquitous Processing}

LIS uses the measured RSSI of a node and its neighbors to determine the area where the non-anchor node could be located. This algorithm is based on a fuzzy system distributed on every anchor node of the network.

According to the algorithm stages, once an anchor node receives a beacon, it estimates the position of the non-anchor nodes. The localization algorithm has been designed to distribute the computation consumption over the network. The area where the non-anchor node could be localized with a certain probability is called the Representative Area. A "sector" is the minimum area formed by three anchor-node neighbors. A Representative Area can be made up of one or more sectors. Anchor nodes must execute the distributed fuzzification algorithm for every surrounding sector. Figure 3 shows an example with five sectors, in which, the fuzzy algorithm is executed five times.

Every anchor node that receives a beacon measures and broadcasts the RSSI level to its anchor neighbors (figure 2.b). In this way, the closest anchor nodes elaborate a table with the RSSI measured by themselves and their anchor neighbors.

The RSSI table is processed by the fuzzy system to evaluate the Representative Area, irrespective of the number of sectors. This area can be formed by the union of one or more sectors (figure 2.c). A sector is considered as a part of the Representative Area if its membership degree is higher than the

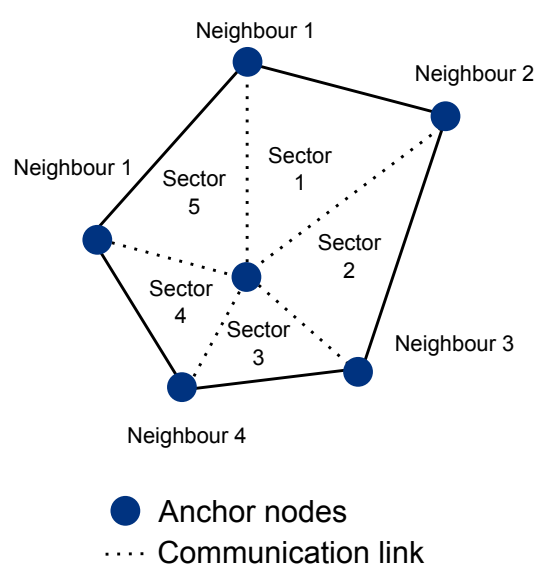

Figure 3: Example of node with 5 neighbours.

threshold. This value is adjusted experimentally. The current simulations show that a threshold of 0.1 manages a good trade-off between the noise immunity and localization performance. The results of the Representative Areas are sent from the anchor nodes to the Base Station to compute the final solution (figure 2.d).

A Representative Area is empty if it does not contain significant sector, i.e. if the membership degree for all of them is lower than the threshold. In this case, to save energy, the result is discarded and the algorithm will finish until the next beacon arrives (figure 4). This is especially important in huge networks, where the energy needed for multi-hop transmissions is high and is a disadvantage of the centralized localization algorithm. This issue is discussed in detail in section 4

\subsubsection{The Fuzzy System Inputs}

RSSI tables represent the signal level received in either the local or the neighbor anchor nodes. Three fuzzy sets qualify the RSSI as High, Medium and Low 
(B.S.)

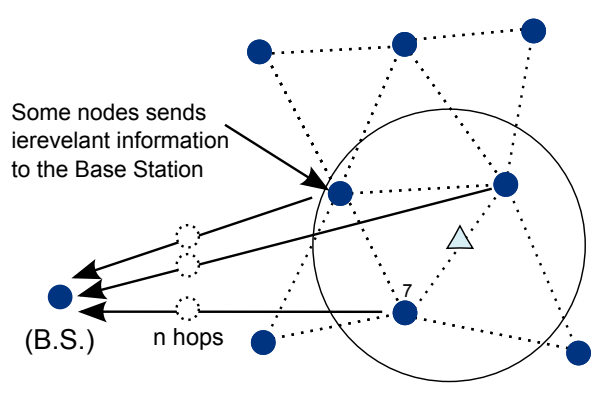

Centraliced Algorithm

In extended networks, redirect messages would waste a lot of energy

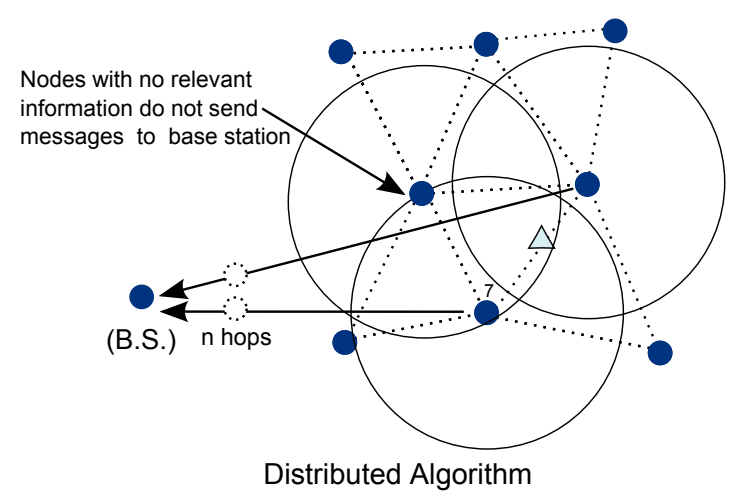

A node decides with our neighbour if it have relevant information

Figure 4: Distributed algorithm would save Power Energy on extended networks.

for each input (figure 5).

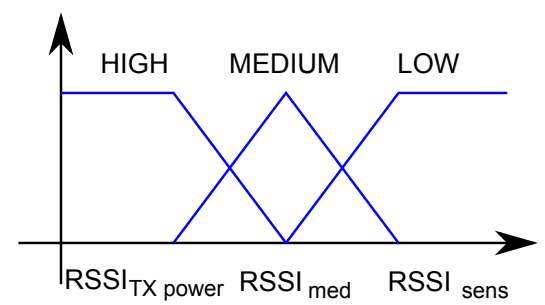

Figure 5: Sets of the fuzzy inputs.

The LOW RSSI fuzzy set is represented by a trapezoid. The maximum membership degree (value 1 ) is assigned if the power falls down the sensibility threshold of the emitter node (RSSI $\left.I_{\text {sens }}\right)$. As the power increases, the membership degree decreases linearly until it reaches zero. Following equation defines this fuzzy set:

$$
\mu(x)=\max \left(\min \left(1, \frac{a-x}{a-b}\right), 0\right)
$$

Where $a=R S S I_{\text {med }}$ and $b=R S S I_{\text {sens }}$.

The medium RSSI fuzzy set is represented by a triangle where the maximum membership degree corresponds to the medium RSSI value (RSSI $I_{\text {med }}$ ). The zero membership is reached for the power RSSI values lower than the sensibility threshold or close to the maximum transmission (RSSI $I_{T X}$ power). In the current study, the medium RSSI value must be computed for every sector using the Friis model equation and assuming the emitter tag is located at the centre. This computation only needs to be executed once because the anchor nodes are located at fixed positions.

$$
\frac{P_{R X}}{P_{T X}}=G_{T X} \cdot G_{R X} \cdot\left(\frac{\lambda}{4 \pi R}\right)^{2}
$$

Where $G_{T X}$ and $G_{R X}$ are the gain of TX and RX antennas, $R$ is the distance between transmitter and receiver and $\lambda$ the wavelength.

The use of Friis is a trade-off between the accuracy and the information required. More realistic models require having more initial information of the environment, a priori unknown, such as the position of the obstacles. The Friis approximation simplifies the problems of the saw-tooth of the disturbances with a smooth function. According to a real scenario, this assumption could not be a good approximation to the reality. However, the errors assumed with this approximation are compensated with the noise immunity of LIS, which assumes the disturbances as noise.

Next expression defines the fuzzy set for MEDIUM RSSI:

$$
\mu(x)=\max \left(\min \left(\frac{x-a}{b-a}, \frac{c-x}{c-b}\right), 0\right)
$$

Where $a=R S S I_{\text {sens }}, \quad b=R S S I_{\text {med }}$ and $c=$ $R S S I_{T X}$ power.

Fuzzy set for HIGH RSSI values is a trapezoid with a lineal increasing from 0 to 1 for the RSSI power values ranging between $R S S I_{\text {med }}$ and $R S S I_{T X}$ power. This fuzzy set is defined by the next expression:

$$
\mu(x)=\max \left(\min \left(\frac{x-a}{b-a}, 1\right), 0\right)
$$

Where $a=R S S I_{\text {med }}, b=R S S I_{T X}$ power.

\subsubsection{The Fuzzy System Outputs}

The Fuzzy System offers an output for each and every sector. The output associated to a sector is a $[0,1]$ ranged value that represents the confidence degree that the tag is actually located in that sector. 


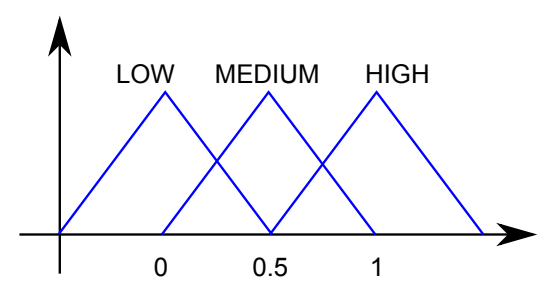

Figure 6: Sets of the fuzzy output.

As figure 6 shows, the LOW output fuzzy set is a triangle with the central point at zero and the corners at -0.5 and 0.5 . Next expression defines this fuzzy set:

$$
\mu(x)=\max \left(\min \left(\frac{x+0.5}{0.5}, \frac{0.5-x}{0.5}\right), 0\right)
$$

The MEDIUM output is represented by a triangle with the central point at 0.5 and corners at 0 and 1 . Mathematically it can be expressed by the following equation:

$$
\mu(x)=\max \left(\min \left(\frac{x}{0.5}, \frac{1-x}{0.5}\right), 0\right)
$$

The HIGH output qualifier is also defined by a triangle with the central point at 1 and the corners at 0.5 and 1.5. This fuzzy set is defined by the next expression:

$$
\mu(x)=\max \left(\min \left(\frac{x-0.5}{0.5}, \frac{1.5-x}{0.5}\right), 0\right)
$$

\subsubsection{Inference Engine}

The inference engine is the Mandani's rules based one with a centroid defuzzification method and a singleton input fuzzificator. The fuzzy engine evaluates the antecedent of every rule by the intersection of the fuzzy inputs, using the minimum function for the AND operator (Eq. 8), and the maximum function for the OR operator (Eq. 9). The implication between the inputs and outputs applies the minimum function.

$$
\begin{gathered}
\operatorname{AND}(a, b)=\min (\mu(a), \mu(b)) \\
O R(a, b)=\max (\mu(a), \mu(b))
\end{gathered}
$$

As mentioned, the rules must be evaluated for every single sector to estimate the confidence degree, taking into account the fuzzy qualifications of RSSI values of either the current sector nodes or the surrounding ones. The rules summed up in Table 1 have been derived from multiple simulations in order to obtain the best trade-off between precision and noise immunity.
Table 1: Rules of the inference engine.

\begin{tabular}{|c|l|c|}
\hline RSSI node & RSSI Neighbours & Output \\
\hline High & All medium & High \\
\hline Low & All low & Low \\
\hline Medium & All medium & High \\
\hline Medium & All low & Low \\
\hline High & All high & Medium \\
\hline Medium & $\begin{array}{l}\text { Medium in current sector } \\
\text { Low in the rest }\end{array}$ & High \\
\hline Medium & $\begin{array}{l}\text { High in any sector except } \\
\text { the current one } \\
\text { Low in the rest }\end{array}$ & Low \\
\hline High & $\begin{array}{l}\text { High in a neighbour of the } \\
\text { current sector } \\
\text { Low in the rest }\end{array}$ & Medium \\
\hline High & $\begin{array}{l}\text { High in a neighbour, except } \\
\text { on the current sector } \\
\text { Low in the rest }\end{array}$ & Low \\
\hline Medium & $\begin{array}{l}\text { Medium in a neighbour of } \\
\text { the current sector. } \\
\text { Low in the rest }\end{array}$ & Medium \\
\hline Medium & $\begin{array}{l}\text { Medium in a neighbour, } \\
\text { except on the current sector } \\
\text { Low in the rest }\end{array}$ & Low \\
\hline
\end{tabular}

\subsection{Cooperative Processing}

The Base Station collects the partial solutions from the anchor nodes, and processes them cyclically as follows:

C1. The Base Station waits for receiving the first partial solution.

C2. On arrival, the partial solution is saved and a timer starts running.

C3. While the timer is running, the next partial solutions are saved in a table as they were received.

C4. When the timer expires, the system will compute the final position as the centroid of all these partial solutions (triangle sectors). The centroid computation of a finite set of points $\vec{P}_{1}, \vec{P}_{2}, \cdots \vec{P}_{N}$ can be simplified as:

$$
\text { Position }=\frac{\sum_{i=1}^{N} \vec{P}_{i}}{N}
$$

The previous algorithm can be easily extended to locating multiple tags, by simply associating a tag identifier to the transmitted beacons. The final estimated position is time stamped and saved in the Base Station to make it accessible throughout the Internet. 


\section{POWER CONSUMPTION OF LIS}

Generally, power consumption is a strong constraint in a WSN application. This is especially true in mobile target tracking (e.g. wild animals in natural environments). Power constraints are higher for non-anchor or mobile nodes. Anchor nodes can recharge their batteries, using systems as such as solar panels. But for non-anchor nodes, mobility and additional constraints like size and weight do not allow use of high capacity batteries or any other alternative power source.

Most node power consumption is caused by radio transmissions. As an example, the Telosb platform consumes $41 \mathrm{~mW}$ in active mode. The microcontroller consumes only $5 \mathrm{~mW}$ and the remainder power consumption is caused by the radio transceiver that requires $38 \mathrm{~mW}$ in the receiver mode and $35 \mathrm{~mW}$ in transmission (Polastre et al., 2005).

It is important to point out that the power consumption is very high either in transmission or in the reception mode. Therefore, to reduce the power consumption it is necessary to reduce the number of exchange messages, and also stop all the node activity enabling low power modes and switch on the radio transceiver. Therefore, a suitable activity manager with hibernation periods is needed. This problem is analysed later.

Figure 7 represents a localization algorithm computed in the non-anchor node.

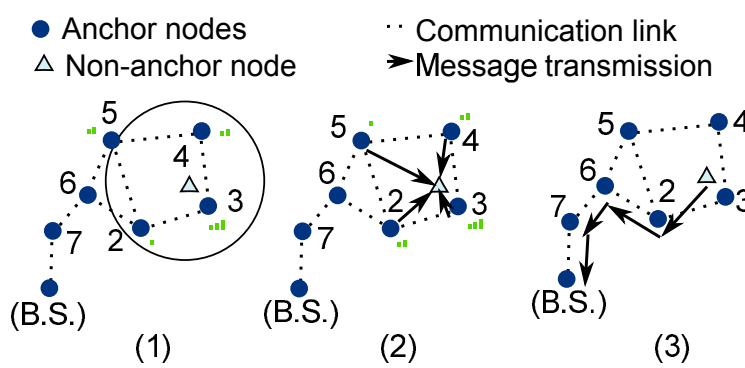

Figure 7: Localization algorithm using the non-anchor node for estimate its positions.

As it can be observed, after the tag node broadcasts a beacon 7.1), it should wait for the response of all the anchor nodes placed in the radio range (7.2). This phase takes a long time because of the number of surrounding nodes and also the collisions. After that, the tag node executes the localization algorithm and delivers the result to the Base Station (7.3). During all of this, the radio transceiver must be in the active state. It wastes a lot of energy and the autonomy is considerably reduced

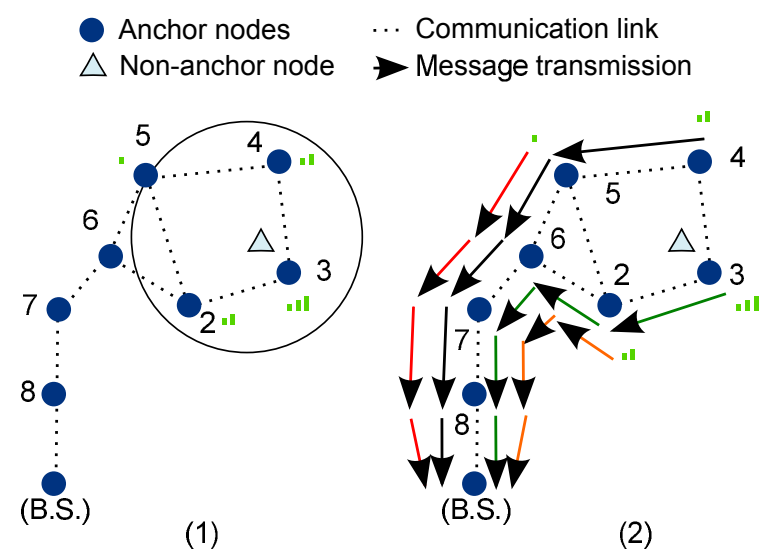

Figure 8: Example of centralized algorithm.

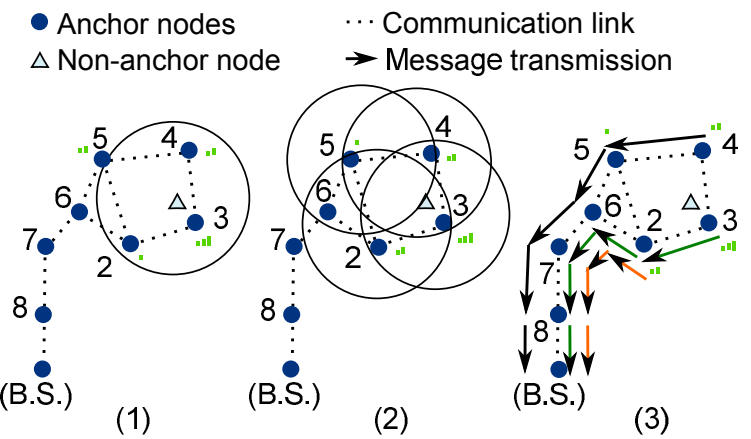

Figure 9: Example of distributed algorithm.

for the non-anchor node. It is in fact, the device with higher energy constraints.

LIS takes this issue into account. Also, that anchor nodes have more power supply resources than the tags. The algorithm has been designed to be executed mainly in the anchor nodes. Furthermore, the radio transceiver of the tag is activated for a short time, just enough to broadcast the beacon. In the remaining period of time, the tag will be in a idle state and its radio transceiver off.

But LIS also reduces the power consumption in anchor nodes. It implements an ubiquitous and distributed algorithm that spreads the localization processing amongst the nodes surrounding the tag. In a centralized-only algorithm, all the information received by the anchor nodes must be delivered to the Base Station (Figure 8). By contrast, proposed algorithm saves power energy because only significant information is delivered (Figure 9).

In the worst case, LIS delivers practically the same number of messages than a centralized algorithm. But for low dense deployments, for example when medium number of nodes that a beacon receives is lower than the medium number of hops necessary to reach the Base Station, the savings is significant. 


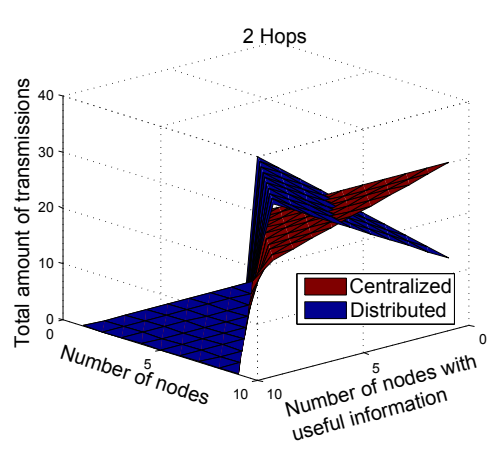

(a)

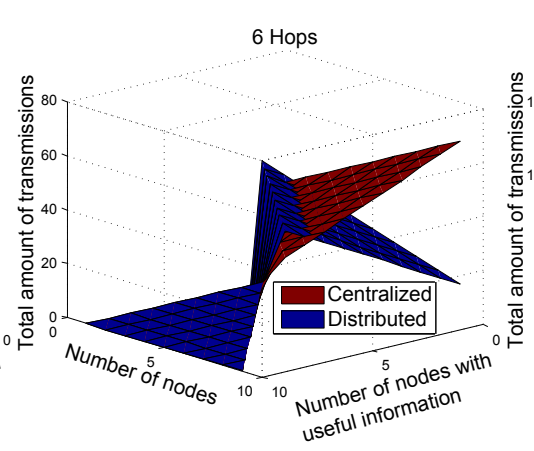

(b)

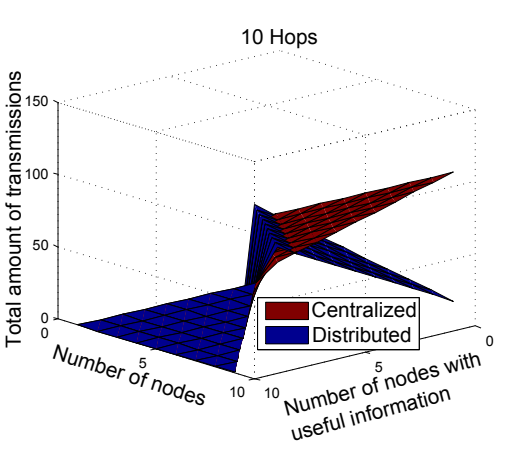

(c)

Figure 10: Messages transmission versus the number of nodes: A) Two hops, B) 6 hops, C) 10 hops.

As a consequence, the energy saved with the distributed algorithm varies with the density and complexity of the networks. Figure 10 shows a study about the number of delivered messages in function of the number nodes with useful information. From this, it can be derived that in case that all the information obtained by the anchor nodes were useful, both methods send practically the same number of messages. But the more number of nodes with useless information, the energy saving performance of the distributed processing increases drastically.

Additional savings can be managed by clustering the networks, and using the cluster heads as the Base Stations, i.e. receiving the processing partial estimations from its cluster nodes. Figure 11 shows this idea.

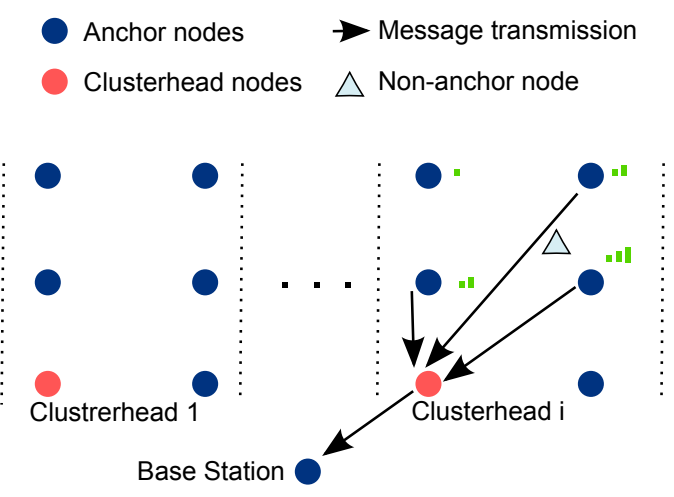

Figure 11: Example of the use of clusters.

For this case, the algorithms must be modified as follows:

S1. Anchor nodes wait for non-anchor node beacons.

S2. The tag node broadcast a beacon.

S3. Receiver anchor nodes measure RSSI, and execute both, fuzzification algorithm and ubiquitous processing for relative and partial positioning.

S4. Anchor nodes send partial solutions to the clusterhead, where the location is finally determined

S5. Clusterhead node executes the cooperative positioning algorithms and delivers the final position to the Base Station.

S6. Base Station executes the same cooperative positioning algorithm than the clusterhead nodes, but using the information delivered from these clusterheads. In this way, if the tag positioning comes from just one clusterhead, this position will be considered as the final solution. But, if it is received from more than one clusterhead, the centroid estimation is applied to all of them.

Determining when the use of clustering saves more energy is not trivial. It depends on the size and complexity of the network. But in general, it is reasonably to think that clustering techniques are better for wide and complex networks.

Additionally, a distributed processing such as the one proposed in this paper, increases Throughput and reduces the response delay, because traffic bottleneck and collisions close to the Base Station are avoided. Distributed processing also spreads computational load over the network. This is especially important for wide networks or with multiple non-anchor nodes.

\section{ACCURACY OF LIS}

The accuracy of LIS versus the classic CL algorithm (Bulusu et al., 2000) was compared using different simulations. The election of centroid as the reference algorithm to compare with is based on the fact that many authors use it in their research studies. Thus, it is possible to obtain a conclusion on the accuracy of LIS not only with the centroid, but also with all the 
other localization techniques that are compared with it in the literature.

The tested network was made up of 25 anchor devices with a radio range of 200 meters in a non-anchor node and with separated anchor nodes also with a radio range of about 200 meters. The anisotropic radiation pattern was assumed. The simulator has been developed in $\mathrm{C}++$. It allows the selection of the radio range, radiation pattern, Gaussian noise, sensibility, network deployment and anchor location. All the parameters, in the tests have been selected to model the Telosb devices and using a Friis propagation model. The results of the simulations are presented in the next subsection.

The use of a simulator for obtaining the accuracy of a localization algorithm is a common tool. It is difficult to compare the localization algorithms in real scenarios, because of the changes of the environment. Thus, these experiments are generally not replicable, even by the author (different days would offer different results). Obtaining unified comparison criteria for the localization algorithm and the necessary parameters to consider in the real experiments is currently an important area of research, which is far from over. However, simulation results are easily reproducible and permit the comparison of the localization algorithms amongst themselves.

\subsubsection{Error vs. Position}

The following experiments include a moving tag into the network. The noise has been neglected and the error is expressed in meters. As figures 12 and 13 show, the maximum end medium errors of LIS algorithm are considerably smaller than the ones estimated with the (CL) Centroid classic algorithm.

Figure 12 is a graphical representations of the errors, which represents the real position and the estimated position of the algorithms in a few analyses. The results are normalized with the radio coverage of the non-anchor nodes that are 200 meters in these tests.

Figure 13 represents the position error, measured as the absolute value of the Euclidean distance between the real and the estimated position. The results are normalized with the non-anchor node radio range. They are obtained by moving the non-anchor node into the quadrant made up by the anchor nodes $7,8,9$ and 12, in a step of 2 meters. The $\mathrm{X}$ axis represents the relative position of the tag into the quadrant, assuming that node 8 is the coordinates origin. These values are normalized with the distance between then nodes (200 meters). The last relative point simulated is [0.5 0.5$]$ because the other three

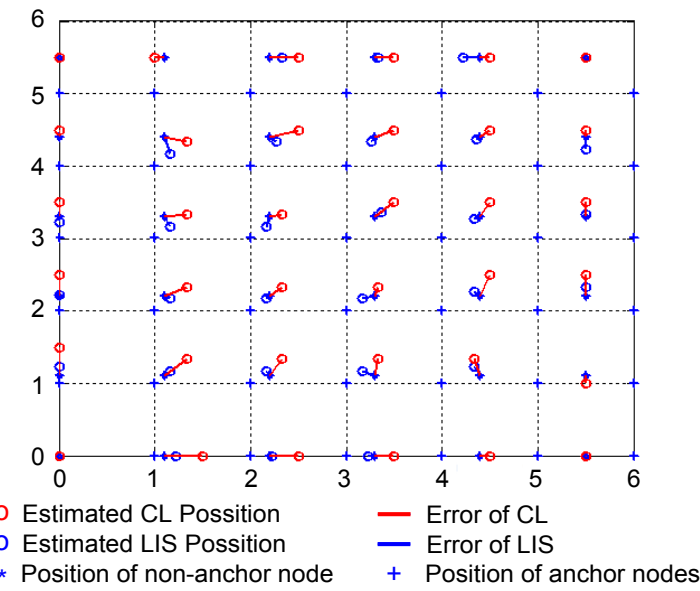

Figure 12: Position error of CL algorithm and LIS algorithm.

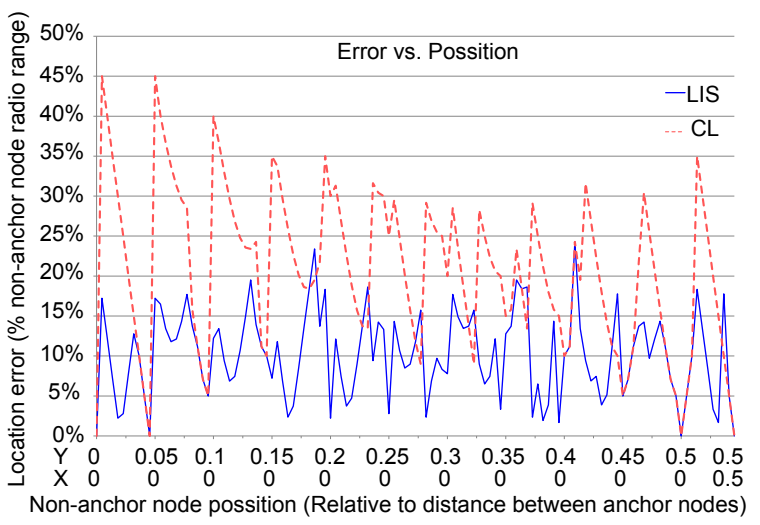

Figure 13: Localization error vs. the position of the non-anchor node.

parts of the quadrant offer symmetrical results than obtained result.

\subsubsection{Error vs. Coverage}

Figure 14 shows the influence of the radio range. As the radio range increases, the number of non-anchor nodes that receive a beacon also increases, and the error decreases. In this scenario the noise has not been considered. The radio range of the non-anchor node simulated is in the [100 m-350 m] range.

Results are the relative error to the distance between the anchor nodes that are separated 200 meters. There are six different points, which are simulated corresponding to the points 1 to 6 represented in the figure, separated by 50 meters. These points are into the quadrant of the nodes 7 , 8,12 and 13 of the network. The rest of the points can be obtained by dividing the quadrant in step of 50 meters, which offers identical results than those of the 
simulated. This is because of the symmetry.

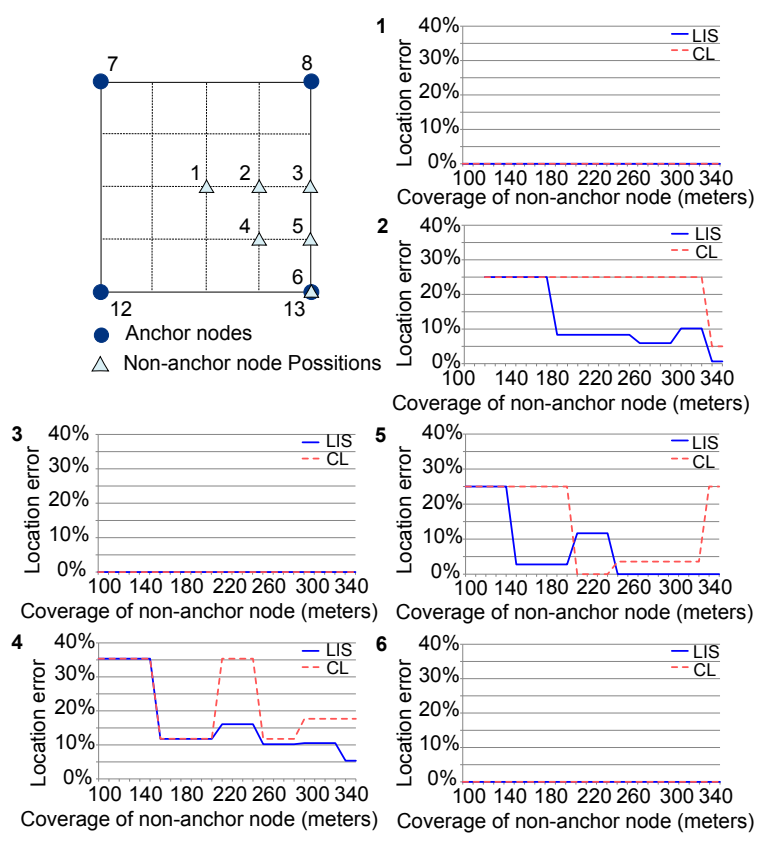

Figure 14: Localization error vs. the coverage radio area of non-anchor node.

In all of the cases, LIS gets smaller or equal errors than the centroid algorithm. Similar results are obtained by fixing the coverage area of the non-anchor nodes and reducing the distance between the anchor devices from the simulated 200 meters. These results are similar to the evaluation of the error versus the node density, which was proposed by the other authors.

As it can be seen in points where there is symmetry between the position of the tags and the anchor nodes (points 1, 3 and 6), the error is always 0 for all the simulated coverage. This is a typical behavior of the centroid, which has low errors in these symmetrical points, but has very bad behavior outside these points. LIS presents a good behavior in all of the simulated points.

With the two algorithms, better accuracy is obtained by increasing the number of anchor nodes that receives the beacon, but with the proposed method, the system tends to lower the errors earlier.

\section{CONCLUSIONS AND FUTURE WORK}

LIS is a new fuzzy algorithm for localization designed to reduce power consumption, especially but not limited to, the tag nodes where the power constraints are higher. LIS filters the useless information after being processed in the anchor nodes. It also implements a hibernation mechanism. All these mechanisms increase the battery autonomy.

LIS has been tested by simulations. The obtained results showed that the proposed method obtains less localization errors than the CL algorithm without higher computation requirements or an extensive use of radio.

The localization system LIS is being applied to locating and tracking of wild animals in natural parks.

\section{ACKNOWLEDGEMENTS}

This research has been supported by the "Consejería de Innovación, Ciencia y Empresa", "Junta de Andalucía", Spain, through the excellence project ARTICA (reference number: P07-TIC-02476) and by the "Cátedra de Telefónica, Inteligencia en la Red", Seville, Spain, through the project ICARO.

The authors would like to thank the Biological Station of the natural park of "Doñana" and the researchers of its Biological Station Centre, for their collaboration and support.

\section{REFERENCES}

Akyildiz, I. F., Su, W., Sankarasubramaniam, Y., and Cayirci, E. (2002). Wireless sensor networks: A survey. Computer Networks, 38(4):393-422.

Antoine-Santoni, T., Santucci, J. F., de Gentili, E., Silvani, X., and Morandini, F. (2009). Performance of a protected wireless sensor network in a fire. analysis of fire spread and data transmission. Sensors, 9(8):5878-5893.

Awad, A., Frunzke, T., and Dressler, F. (2007). Adaptive distance estimation and localization in wsn using rssi measures. In 10th Euromicro Conference on Digital System Design Architectures, Methods and Tools, DSD 2007, pages 471-478. Sponsors: Drager; Conference code: 72733; Cited By (since 1996): 11.

Boukerche, A., Oliveira, H. A. B. F., Nakamura, E. F., and Loureiro, A. A. F. (2008). Secure localization algorithms for wireless sensor networks. IEEE Communications Magazine, 46(4):96-101. Cited By (since 1996): 11.

Bulusu, N., Heidemann, J., and Estrin, D. (2000). Gps-less low-cost outdoor localization for very small devices. IEEE Personal Communications, 7(5):28-34.

Chiang, S. Y. and Wang, J. L. (2009). Localization in wireless sensor networks by fuzzy logic system. In 13th International Conference on Knowledge-Based and Intelligent Information and Engineering Systems, KES 2009, volume 5712 LNAI, pages 721-728. 
Chung, W. Y., Lee, Y. D., and Jung, S. J. (2008). A wireless sensor network compatible wearable u-healthcare monitoring system using integrated ecg, accelerometer and spo2. In 30th Annual International Conference of the IEEE Engineering in Medicine and Biology Society, EMBS'08, pages 1529-1532.

Gao, G. Q. and Lei, L. (2010). An improved node localization algorithm based on dv-hop in wsn. In Northeastern University, volume 4, pages 321-324.

He, T., Krishnamurthy, S., Stankovic, J. A., Abdelzaher, T., Luo, L., Stoleru, R., Yan, T., Gu, L., Hui, J., and Krogh, B. (2004). Energy-efficient surveillance system using wireless sensor networks. MobiSys 2004 - Second International Conference on Mobile Systems, Applications and Services, pages 270-283.

Polastre, J., Szewczyk, R., and Culler, D. (2005). Telos: enabling ultra-low power wireless research. In Information Processing in Sensor Networks, 2005. IPSN 2005. Fourth International Symposium on, pages $364-369$.

Rajaee, S., Almodarresi, S. M. T., Sadeghi, M. H., and Aghabozorgi, M. (2008). Energy efficient localization in wireless ad-hoc sensor networks using probabilistic neural network and independent component analysis. In 2008 International Symposium on Telecommunications, IST 2008, pages 365-370.

Riquelme, J. A. L., Soto, F., Suardáz, J., Sánchez, P., Iborra, A., and Vera, J. A. (2009). Wireless sensor networks for precision horticulture in southern spain. Computers and Electronics in Agriculture, 68(1):25-35

Rong, P. and Sichitiu, M. L. (2006). Angle of arrival localization for wireless sensor networks. In Sensor and Ad Hoc Communications and Networks, 2006. SECON '06. 2006 3rd Annual IEEE Communications Society on, volume 1, pages 374-382.

Tubaishat, M., Peng, Z., Qi, Q., and Yi, S. (2009). Wireless sensor networks in intelligent transportation systems. Wireless Communications and Mobile Computing, 9(3):287-302.

Wu, S. H. and Zhang, N. T. (2007). Two-step toa estimation method for uwb based wireless sensor networks. Ruan Jian Xue Bao/Journal of Software, 18(5):1164-1172.

Xiufang, F., Zhanqiang, G., Mian, Y., and Shibo, X. (2008). Fuzzy distance measuring based on rssi in wireless sensor network. In Proceedings of 2008 3rd International Conference on Intelligent System and Knowledge Engineering, ISKE 2008, pages 395-400.

Yick, J., Mukherjee, B., and Ghosal, D. (2008). Wireless sensor network survey. Computer Networks, 52(12):2292-2330. 